American Journal of Biochemistry and Biotechnology 1 (2): 85-89, 2005

ISSN 1553-3668

(C) 2005 Science Publications

\title{
Biosensor Based on Xanthine Oxidase for Monitoring Hypoxanthine in Fish Meat
}

\author{
Helena Shizuko Nakatani, Luciane Vieira dos Santos, Cristiano Peralta Pelegrine, Sandra Terezinha \\ Marques Gomes, Makoto Matsushita, Nilson Evelázio de Souza and Jesui Vergilio Visentainer \\ Department of Chemistry, State University of Maringa, Av. Colombo, 5790 \\ CEP 87020-900 Maringa, Parana State, Brazil
}

\begin{abstract}
An amperometric enzyme electrode for the detection of hypoxanthine in fish meat is described. The hypoxanthine sensor was prepared from xanthine oxidase immobilized by covalent binding with glutaraldehyde and bovine serum albumin on a nafion-coated platinum disc electrode. The responses for hypoxanthine were obtained in $0.1 \mathrm{~mol} \mathrm{~L}^{-1}$ phosphate buffer $(\mathrm{pH} 7.0)$, at potential of $600 \mathrm{mV}$ vs $\mathrm{Ag} / \mathrm{AgCl}$. This biosensor provides a linear response for hypoxanthine in the concentration range of $2.00 \times 10^{-6}$ $1.85 \times 10^{-4} \mathrm{~mol} \mathrm{~L}^{-1}(\mathrm{r}=0.9989)$. The response time is less than $30 \mathrm{~s}$ for the useful response range and the lifetime of this electrode was excellent for at least ten days without loss of the response. The sensor can be applied to the determination of hypoxanthine in fish meat and it is effective for eliminating interferants from coexisting substances in the samples. Application of the biosensor technique in the field of quality control is promising due to their inherent specificity, simplicity and quick response.
\end{abstract}

Key words: Biosensors, enzyme-electrodes, hypoxanthine, xanthine oxidase, fish

\section{INTRODUCTION}

Estimation of fish meat freshness is important for the food industries for the manufature of high quality products. After the death of a fish, the decomposition of ATP (adenosine triphosphate) in the fish meat sets in and ADP (adenosine di phosphate), AMP (adenosine 5' phosphate), IMP (inosine 5' phosphate), HxR (inosine), Hx (hypoxanthine), X (xanthine) and $U$ (uric acid) ${ }^{[1]}$. Whereas IMP is one of the major contributing factors to the pleasant flavour of fresh fish, it's degradation product hypoxanthine imparts the bitter "off-taste"[2]. In this degradation process, quantification of hypoxanthine can be used as an indication of the fish freshness.

Various methods ${ }^{[1,3-5]}$, including anion-exchange chromatography, thin layer chromatography, precipitation and capillary electrophoresis, have been proposed for the hypoxanthine determination. In general, these methods are complicated and time consuming.

Highly specific analytical techniques have been developed by coupling immobilized redox enzymes with electrochemical sensors. In comparison with other analytical techniques, such sensors possess simplicity of operation and the substrate selectivity of the enzymes. In addition, they are inexpensive since a small amount of immobilized enzyme can be used for a large number of analytical determinations ${ }^{[6,7]}$.

Amperometric sensors using immobized xanthine oxidase have been reported and have shown effectiveness and selectivity for the measurement of hypoxanthine ${ }^{[8-11]}$.
Significant developments in biosensors for fish quality measurement started in the 1980 s, as recently reviewed by Venugopal $^{[12]}$. Pioneering work was carried out by Watanabe's group to measure nucleotide concentrations to evaluate fish freshness ${ }^{[13-15]}$. Shen, Yang, \& Peng ${ }^{[16]}$ developed a simpler method that uses a xanthine oxidase electrode. The enzyme was immobilized on a silk membrane, onto which a platinum disc and copper wire were attached. This device was highly sensitive to Hypoxanthine (Hx) levels in fish and could therefore be used to assess fish freshness.

Metal as platinum have been used for electrochemical electrodes due to their excellent electrical and mechanical properties ${ }^{[8]}$.

Operation of an electrochemical biosensor requires successful enzyme immobilization on the electrode surface. In the search for suitable matrices for enzyme immobilization there has been growing interest in the attachment of the enzyme to the solid electrolyte Nafion. The method of membrane formation involves simply dipping the electrode into the polyelectrolyte solution and allowing the solvent to evaporate. The resulting membranes possess both high adhesion to the surface and low swelling in aqueous media. In addition, the polyelectrolyte membrane stabilizes the ionic strength at the electrode surface, essential for sensor applications. Nafion exhibits a reduced permeability to negatively charged substances. Hence by using an additional Nafion membrane one can improve the sensor selectivity and virtually eliminate the influence of reductants ${ }^{[17]}$.

Corresponding Author: Nilson Evelázio de Souza, Department of Chemistry, State University of Maringa, Av. Colombo, 5790, CEP 87020-900 Maringa, Parana State, Brazil, Fax: + 55-44-263-5784 
The aim of the present work was to develop a amperometric hypoxanthine biosensor, based on xanthine oxidase immobilized on a Nafion-coated platinum disc electrode. Coating of Nafion has successfully been used to eliminate the interference of uric acid and ascorbic acid in the determination of hypoxanthine.

\section{MATERIALS AND METHODS}

Reagents: Xanthine oxidase (EC 1.1.3.22, from buttermilk, Grade I), xanthine, hypoxanthine, ascorbic acid, uric acid, glutaraldehyde (50\% aqueous solution), bovine serum albumin (lyophilized powder) and cellulose acetate were obtained from Sigma. Nafion 5\% was obtained from Aldrich. Other chemicals were of analytical grade.

Electrochemical instrument: Amperometric measurements were performed on a EG \& G Princeton Applied Research Model 263 potentiostat with M270 software system, using an electrochemical cell with three electrodes. $\mathrm{An} \mathrm{Ag} / \mathrm{AgCl}$ electrode was used as reference, a Pt wire as the auxiliary and the working enzyme electrode was the Nafion-coated platinum disc electrode.

Electrochemical instrument: Amperometric measurements were performed on a EG \& G Princeton Applied Research Model 263 potentiostat with M270 software system, using an electrochemical cell with three electrodes. $\mathrm{An} \mathrm{Ag} / \mathrm{AgCl}$ electrode was used as reference, a Pt wire as the auxiliary and the working enzyme electrode was the Nafion-coated platinum disc electrode.

Electrode preparation: The enzyme electrode was constructed by cross-linking the xanthine oxidase and bovine serum albumin (BSA) with glutaraldehyde on the Nafion-coated surface of the platinum disk $(3 \mathrm{~mm}$ diameter), freshly cleaned with diluted nitric acid, alcohol and water, sequentially.

The preparation steps of this hypoxanthine sensor were:

(a) Deposition of the Nafion film coating: $5 \mu \mathrm{L}$ of $5 \%$ Nafion solution was pipetted on the electrode surface. The film was allowed to dry during 1 hour.

(b) Immobilization of the enzyme: $15 \mu \mathrm{L}$ of xanthine oxidase (XOD) (25 U), $5 \mu \mathrm{L}$ of $10 \%(\mathrm{w} / \mathrm{v})$ BSA and $5 \mu \mathrm{L}$ of $5 \%(\mathrm{v} / \mathrm{v})$ glutaraldehyde was added and mixed homogeneously. $10 \mu \mathrm{L}$ of this resulting enzyme solution was used and drop it onto the surface of the Nafion-film electrode and dried in a dessecator at room temperature for 3 hours.

Coating of sensor: The electrode was modified by immersing in $5 \mu \mathrm{L}$ of $2 \%$ cellulose acetate solution.
This film was allowed to dry in air for 10 hours. The enzyme electrode was dipping for the conditioning at $5^{\circ} \mathrm{C}$ in a phosphate buffer $\left(0.1 \mathrm{~mol} \mathrm{~L}^{-1}, \mathrm{pH} 7.0\right)$ prior to use.

Electrochemical measurements: Initially the current was monitored continuously in buffer solution until it reached the steady state (from 5 to $10 \mathrm{~min}$ ). After that, successive additions of hypoxantine standard solution were added into to the cell, stirring the solution for a few seconds before monitoring the current until it reached the steady state. The anodic current between the initial and the maximum current was used to quantify the hypoxanthine concentration. Current outputs were measured at $+600 \mathrm{mV}$ vs $\mathrm{Ag} / \mathrm{AgCl}$. The enzyme electrode was stored at $5^{\circ} \mathrm{C}$ in a phosphate buffer $\left(0.1 \mathrm{~mol} \mathrm{~L}^{-1}, \mathrm{pH}\right.$ 7.0) when not in use.

Determination of hypoxanthine in fish meat: Methods were proposed for the preparation of the fish sample ${ }^{[15,18]}$. However, the proposed method by Watanabe et al. ${ }^{[15]}$, was selected because the simplicity. Fish meat sample $(5 \mathrm{~g})$ was homogenized in $15 \mathrm{~mL}$ distilled water. The homogenate was then filtered through a membrane filter and then distilled water was added to the filtrate producing a total volume of $25.00 \mathrm{~mL}$ of homogenized sample solution. The sample solution was used immediately. The developed sensor was then used to measure the $\mathrm{Hx}$ in the sample. An aliquot of the extract was transferred into an electrochemical cell containing phosphate buffer solution $\left(0.1 \mathrm{~mol} \mathrm{~L}^{-1}, \mathrm{pH} 7.0\right)$ and the electrochemical measurements was made in potential of $600 \mathrm{mV}$ vs $\mathrm{Ag} / \mathrm{AgCl}$.

Determination of hypoxanthine was performed by applying the standard additions method, which involved the addition of successive aliquots of hypoxanthine standard solution.

\section{RESULTS AND DISCUSSION}

In this study, the corresponding enzymatic reaction involved in the membrane is:

Hypoxanthine $+2 \mathrm{O}_{2} \stackrel{\text { Xanthine oxidase }}{\longrightarrow}$ Uric acid $+2 \mathrm{H}_{2} \mathrm{O}_{2}$

The amperometric signal employed for monitoring the oxidation of hypoxanthine catalyzed by XOD was accomplished by means of the electrochemical oxidation of the hydrogen peroxide generated as a product in the enzymatic reaction. A potential of 600 $\mathrm{mV}$ was selected for the amperometric monitoring of the reaction.

To evaluate the performance of the enzyme electrode, several parameters, including immobilization conditions and amounts, $\mathrm{pH}$ and temperatures 
dependence, interference and stability of the electrode were investigated.

Effects of the $\mathrm{pH}$ and temperatures on the response of the sensor: The response of a biosensor is often $\mathrm{pH}-$ and temperature-dependent. Normally, the current output from a biosensor, as a function of $\mathrm{pH}$, will pass through a maximum. This existence of an optimum $\mathrm{pH}$ is due to the amphoteric nature of the amino acids that make up the enzyme, particularly those that are responsible for substrate binding. Protonation can substantially alter the rates of formation and decomposition of the enzyme-substrate complex.

The effect of the $\mathrm{pH}$ value in the test medium on the current measurements of hypoxanthine gives a maximum response between $\mathrm{pH} 6.0$ and 7.5 and was observed that when the $\mathrm{pH}$ is less than 6.0 or larger than 7.5 an amperometric current decrease. A working $\mathrm{pH}$ of 7.0 was selected for subsequent work.

The temperature dependence of these sensors is similar to that of the free enzyme systems, conforming to the general trend of increasing current responses with increasing temperature.

The response to temperature was observed that an increasing current response with increasing temperature between $25.0-35.0^{\circ} \mathrm{C}$. However, the determination using biosensors in highly temperature exhibit a decreasing of oxygen in the solution and the inactivation of the enzyme. As a compromise between sensitivity and stability, a working temperature of the $25.0 \pm 0.1^{\circ} \mathrm{C}$ was chosen in this study.

Analytical characteristics of the calibration curve for hypoxanthine: Under the optimized working conditions described above, a calibration curve was constructed for hypoxanthine. The response time for this biosensor was very show, reaching $95 \%$ of its maximum response is less than $30 \mathrm{~s}$. The typical amperometric curve obtained for the successive additions of $\mathrm{Hx}$ at an applied potential of $600 \mathrm{mV}$ is show in Fig. 1. It can be observed an increasing anodic current response for each successive additions of hypoxanthine solutions. A calibration curve typical of enzymatic systems was obtained for the steady-state current versus the hypoxanthine concentration with a range of linearity $2.00 \times 10^{-5}-1.90 \times 10^{-4} \mathrm{~mol} \mathrm{~L}^{-1}$, by fitting data points by least-squares regression of $\Delta \mathrm{i}_{\mathrm{p}}=$ $8,095+16,91 \mathrm{C}(\mathrm{r}=0,9989)$ and a loss of linearity for higher hypoxanthine concentrations. The relative standard deviation for six determinations for $2.00 \times 10^{-5} \mathrm{~mol} \mathrm{~L}^{-1} \mathrm{Hx}$ was $2.9 \%$. The minimum detectable concentration of $\mathrm{Hx}$ was estimated to be $5.28 \times 10^{-7} \mathrm{~mol} \mathrm{~L}^{-1}$.

Seletivity and stability of the sensor: The effect of the presence of electrochemically active interferants which may be present in samples for hypoxanthine determination, such as ascorbic acid and uric acid, was

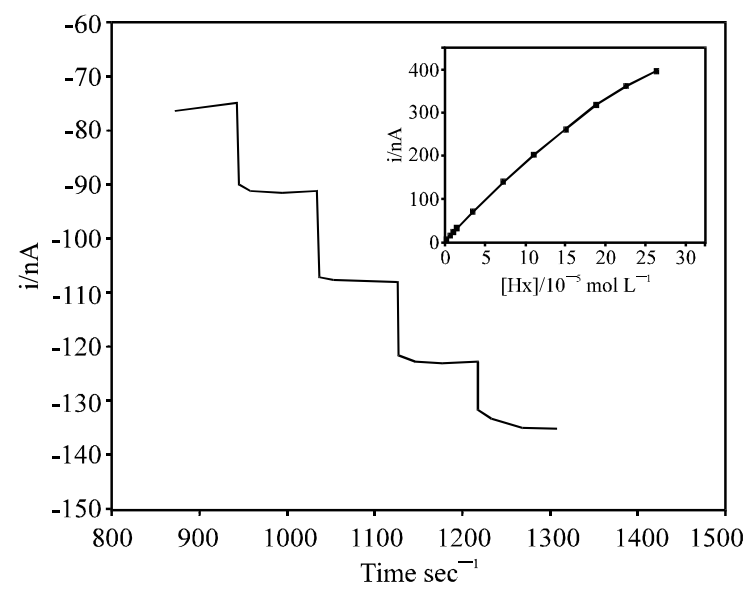

Fig 1: Amperometric curve for hypoxanthine (potential applied $600 \mathrm{mV}$ vs the $\mathrm{Ag} / \mathrm{AgCl}$ ). Each step represents $10 \mu \mathrm{L}$ addition of $0.01 \mathrm{~mol} \mathrm{~L} \mathrm{~L}^{-1}$ hypoxanthine solution in $5.0 \mathrm{~mL}$ of phosphate buffer $\left(0.1 \mathrm{~mol} \mathrm{~L}^{-1}, \mathrm{pH} 7.0\right)$. Inset: calibration curve for enzyme electrode for successive additions of hypoxanthine solution

checked under the experimental conditions specified. These substances, can be oxidized with the generation of a current in the potential selected. Permeable membranes such as Nafion and cellulose acetate covering the platinum electrode were used to reduce the interference effect.

The use of Nafion film in the platinum electrode yields enhanced selectivity towards hydrogen peroxide of the end-products generated by enzymatic reaction.

The selectivity and stability was enhanced by coating of the enzymatic membrane with cellulose acetate membrane. This to make possible one major number of determinations and the increase in the lifetime of biosensor and when absent a sensitiviy decrease due the loss enzymatic material and no of the activity was observed.

The stability of the sensor was examined by measuring the sensor response at a fixed hypoxanthine concentration of $3.98 \times 10^{-5} \mathrm{~mol} \mathrm{~L}^{-1}$. These results show that the current response (relative enzyme activity) remain the same after 80 measurements for over a period of ten days.

The determinations in a phosphate buffer

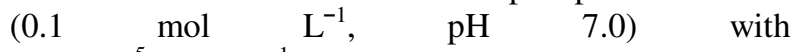
$3.98 \times 10^{-5} \mathrm{~mol} \mathrm{~L}^{-1} \mathrm{Hx}$, the presence of these test compounds in concentration 10 times of $\mathrm{Hx}$ did not cause any observable changes in the current response for the sensor.

The biosensor can be applied to the determination of hypoxanthine in fish meat and it is effective for eliminating interferants from coexisting substances in the samples.

Determination of hypoxanthine in fish meat samples: The performance of the biosensor for the 
analysis of real fish samples was tested by determining the Hx content in samples of Tilapia fish (Oreochromis niloticus) acquired in fish pond and in market. Determination of fish quality is reflected directly by increase in the Hx concentration ${ }^{[8,13,19]}$.

Table 1: Determination of hypoxanthine in fish samples using proposed sensor

\begin{tabular}{lc}
\hline Samples ${ }^{1}$ & Hypoxanthine $\left[\mu \mathrm{mol} \mathrm{g}^{-1}\right]^{2}$ \\
\hline A1 & $4.23 \pm 0.12$ \\
A2 & $16.38 \pm 0.68$ \\
B1 & $2.76 \pm 0.10$ \\
B2 & $8.40 \pm 0.37$ \\
\hline${ }^{1} \mathrm{~A} 1=$ fresh fish; A2 and B2=fish stored in room temperature for 48 \\
hours; B1=fish stored in room temperature for 24 hours \\
${ }^{2}$ Values obtained by application standard-addition method, average \\
value of triplicates
\end{tabular}

Table 2: Recovery (\%) obtained for hypoxanthine in fish samples

\begin{tabular}{|c|c|c|c|c|}
\hline Samples $^{1}$ & $\begin{array}{l}\text { Hx Added }^{2} \\
{\left[\mu \mathrm{mol} \mathrm{L}^{-1}\right]}\end{array}$ & $\begin{array}{l}\text { Hx Expected } \\
{\left[\mu \mathrm{mol} \mathrm{L}^{-1}\right]}\end{array}$ & $\begin{array}{l}\text { Hx Found } \\
{\left[\mu \mathrm{mol} \mathrm{L}^{-1}\right]}\end{array}$ & $\begin{array}{l}\text { Recovery } \\
\%\end{array}$ \\
\hline \multirow[t]{2}{*}{$\overline{\mathrm{A} 1}$} & 0 & & 8.36 & \\
\hline & 9.89 & 18.25 & 19.79 & 108.4 \\
\hline \multirow[t]{2}{*}{ A2 } & 0 & & 19.53 & \\
\hline & 9.93 & 29.46 & 30.54 & 103.7 \\
\hline \multirow[t]{2}{*}{ B2 } & 0 & & 16.63 & \\
\hline & 9.89 & 26.52 & 27.97 & 105.5 \\
\hline
\end{tabular}

${ }^{1}$ samples indicated in the Table 1

${ }^{2}$ standard solution of hypoxanthine added

${ }^{3}$ hypoxanthine concentration expected (sample + standard)

${ }^{4}$ hypoxanthine concentration found

${ }^{5}$ recovery by standard addition method

Table 3: Determination of hypoxanthine in fish samples after different periods of storage

\begin{tabular}{lcl}
\hline Samples $^{1}$ & $\begin{array}{c}\text { Time of storage } \\
(\mathrm{days})^{2}\end{array}$ & $\begin{array}{l}\text { Hypoxanthine } \\
\left.[\mu \mathrm{mol} \mathrm{g}]^{-1}\right]^{3}\end{array}$ \\
\hline $\mathrm{A}$ & 0 & $0.877 \pm 0.026$ \\
$\mathrm{~A}$ & 2 & $1.314 \pm 0.031$ \\
$\mathrm{~B}$ & 7 & $3.944 \pm 0.128$ \\
$\mathrm{~A}$ & 14 & $1.584 \pm 0.072$ \\
$\mathrm{~A}$ & & $1.843 \pm 0.048$ \\
$\mathrm{~A}$ & 30 & $1.904 \pm 0.062$ \\
$\mathrm{~B}$ & 60 & $8.332 \pm 0.389$ \\
$\mathrm{~A}$ & $4.052 \pm 0.135$ \\
${ }^{1} \mathrm{~A}=$ fish pond; B=fish market \\
${ }^{2} \mathrm{After}$ the killed stored in refrigeration at $-18^{\circ} \mathrm{C}$ \\
${ }^{3}$ Values obtained by application standard-addition method, average \\
value of triplicates
\end{tabular}

The Table 1 shows results obtained in fish samples stored at different periods and room temperature and then content of hypoxanthine was determined by standard addition method. As can be observed the amount of hypoxanthine increased with the time of sample storage. The analysis were carried out in triplicate. The effectiveness of the method can then be established by evaluating the extent of recovery of the added quantify of hypoxanthine. Table 2 summarizes the tests results, which show good recovery $(104 \%$ to $108 \%)$.

Table 3 summarizes the results for two fish samples, which show that Hx accumulates with increase of storage time. The increase in Hx accumulation with storage time is expected consequences. These results indicate the suitability of the experimental $\mathrm{Hx}$ biosensor to provide expedient measurements in real fish samples.

\section{CONCLUSION}

The immobilization of xanthine oxidase onto an electrode presents several advantages. The amount of enzyme used in the analysis is significantly lowered. The analysis time can usually be significantly shortened. Biosensors offer advantages as alternatives to a conventional methods due to their inherent specificity, simplicity and quick response.

Determination of the freshness of fish meat can be accomplished by analyzing the Hx. The sensor can be applied to the determination of hypoxanthine in fish meat and it is effective for eliminating interferants from coexisting substances in the samples.

The results obtained are evidence that the present sensor is a very effective analysis system and provides a simple and rapid method for the determination of $\mathrm{Hx}$ in fish samples. Under optimized conditions the proposed sensor presented good stability, making possible in a next step the coupling of this proposed biosensor in a flow system.

\section{ACKNOWLEDGEMENT}

The authors thanks CNPq for financial support.

\section{REFERENCES}

1. Kassemsarn, B.O., P. Sang, J. Murray and N.R. Jones, 1963. Nucleotide degradation in the muscle of iced haddock (Gadus aeglefinus), lemon sole (Pleuronectes microcephalus) and plaice (Leuronectes platessa). J. Food Sci., 28: 28-37.

2. Mulchandani, A., J.T.H. Luong and K.B. Male, 1989. Development and application of a biosensor for hypoxanthine in fish extract. Anal. Chim. Acta, 221: 215-222.

3. Jones, N.R. and J. Murray, 1962. Degradation of adenine and hypoxanthine- nucleotide in the muscle of chill-stored trawled cod (Gadus callarias). J. Sci. Food Agric., 13: 475-480.

4. Tarr, H.L.A., 1966. Post-mortem changes in glycogen, nucleotides, sugar phosphates and sugars in fish muscles- A review. J. Food Sci., 31: 846854.

5. Nguyen, A.L., J.H.T. Luong and C. Masson, 1990. Determination of nucleotides in fish tissues using capillary electrophoresis. Anal. Chem., 62: 24902493.

6. Mello, L.D. and L.T. Kubota, 2002. Review of the use of biosensors as analytical tools in the food and drink industries. Food Chem., 77: 237-256. 
7. Sharma, S.K., N. Sehgal and A. Kumar, 2003. Biomolecules for development of biosensors and their applications. Cur. Applied Phys., 3: 307-316.

8. Yao, T., 1993. Enzyme electrode for the successive detection of hypoxanthine and inosine. Anal. Chim. Acta, 281: 323-326.

9. Park, I.S., Y.J. Cho and N. Kim, 2000. Characterization and meat freshness application of a serial three-enzyme reactor system measuring ATP-degradative compounds. Anal. Chim. Acta, 404: 75-81.

10. Pei, J. And X.Y. Lee, 2000. Xanthine and hypoxanthine sensors based on xanthine oxidase immobilized on a $\mathrm{CuPtCl}_{6}$ chemically modified electrode and liquid chromatography electrochemical detection. Anal. Chim. Acta, 414: 205-213.

11. Wang, L. and Z.B. Yuan, 2004. Direct electrochemistry of xanthine oxidase at a gold electrode modified with single-wall carbon nanotubes. Anal. Sci., 20: 635-638.

12. Venugopal, V., 2002. Biosensors in fish production and quality control. Biosens. Bioelectron., 17: 147-157.

13. Watanabe, E., K. Ando, I. Karube, H. Matsuoka and S. Suzuki, 1983. Determination of hypoxanthine in fish meat with an enzyme sensor. J. Food Sci., 48: 496-500.
14. Watanabe, E., S. Tokimatsu, K. Toyama, I. Karube, H. Matsuoka and S. Suzuki, 1984. Simultaneous determination of hypoxanthine, inosine, inosine-5' -phosphate and adenosine-5'phosphate with a multielectrode enzyme sensor. Anal. Chim. Acta, 164: 139-146.

15. Watanabe, E., A. Nagumo, M. Hoshi, S. Conagaya and M. Tanaka, 1987. Microbial sensors for the detection of fish freshness. J. Food Sci., 52: 592595.

16. Shen, L. Q., L.J. Yang and T.Z. Peng, 1996. Amperometric determination of fish freshness by a hypoxantine biosensor. J. Sci. Food Agric., 70: 298-302.

17. Turner, A. P. F., I. Karube and G. Wilson, 1987. Biosensors- Fundamentals and Applications. Oxford University Press, Oxford.

18. Analytical Methods, $1979 . \quad$ Committee recommended general methods for the examination of fish and fish products. Analyst, 104: 434-450.

19. Niu, J. and J.Y. Lee, 2000. Bulk-modified amperometric biosensors for hypoxanthine based on sol-gel technique. Sensor Actuat. B Chem., 62: 190-198. 